\title{
Generalization of cosmological attractor approach to Einstein-Gauss-Bonnet gravity
}

\author{
Ekaterina O. Pozdeeva ${ }^{\mathrm{a}}$ (D) \\ Skobeltsyn Institute of Nuclear Physics, Lomonosov Moscow State University, Leninskie Gory 1, 119991 Moscow, Russia
}

Received: 26 May 2020 / Accepted: 21 June 2020 / Published online: 8 July 2020

(C) The Author(s) 2020

\begin{abstract}
We construct models with the Gauss-Bonnet term multiplied by a function of the scalar field leading to an inflationary scenario. The consideration is related to the slowroll approximation. The cosmological attractor approach gives the spectral index of scalar perturbations which is in a good agreement with modern observation and allows for variability of the tensor-to-scalar ratio. We reconstruct models with variability of parameters, which allows one to reproduce cosmological attractor predictions for inflationary parameters in an approximation of the leading order of $1 / \mathrm{N}$ in Einstein-Gauss-Bonnet gravity.
\end{abstract}

\section{Introduction}

The solution to the problems of horizon, smoothness, flatness and monopoles, which are related with the hot big-bang model, was proposed by the introduction of inflation [1-10].

The $R^{2}$ inflationary predictions $[11,12]$ in the leading approximation in terms of the inverse e-folding number $1 / N$ for the spectral index $n_{s}$ and the tensor-to-scalar ratio $r$ :

$n_{s} \simeq 1-\frac{2}{N}, \quad r \simeq \frac{12}{N^{2}}$,

are in good agreement with Planck 2018 data $^{1}$ [13].

\footnotetext{
1 There exist two variants for the interpretation of the relation between the time derivative and the e-folding number derivative: (1) $\frac{\mathrm{d}}{\mathrm{d} t}=$ $H \frac{\mathrm{d}}{\mathrm{d} N_{e}}$ and (2) $\frac{\mathrm{d}}{\mathrm{d} t}=-H \frac{\mathrm{d}}{\mathrm{d} N}$.

In the case of the first type formulation, the inflation interval in the efolding formulation is $-65<N_{e}<0$.

In the case of the second type formulation, the inflation interval in the efolding formulation is $0<N<65$. The second formation was applied in the cosmological attractor approximation [17-19] and we follow the second formulation with $N=-\ln (a)$.
}

\footnotetext{
a e-mail: pozdeeva@www-hep.sinp.msu.ru (corresponding author)
} URL: https://publons.com/researcher/2732525/ekaterinao-pozdeeva/
The inflationary scenario motivated by the Standard Model of particles physics, Higgs-driven inflation [14-16], leads to the same prediction. Higgs-driven inflation belongs to the class of cosmological attractors [17-19], which generalizes the prediction (1).

The cosmological attractor models predict the same values of observable parameters $n_{\mathrm{S}}$ and $r$ in the leading $1 / N$ approximation:

$n_{s} \simeq 1-\frac{2}{N+N_{0}}, \quad r \simeq \frac{12 C_{\alpha}}{\left(N+N_{0}\right)^{2}}$,

where $C_{\alpha}$ and $N_{0} \ll 60$ are constants.

The Higgs-driven inflation was generalized to multi-field inflationary scenarios [20-22], for which the cosmological attractor approximation is appropriate [23,24].

At present, the interest in inflationary scenarios in the cosmological models with Gauss-Bonnet term is growing [25-35]. In the present paper, we construct a gravity model with the Gauss-Bonnet term multiplied by a function of a scalar field which allows one to reconstruct expressions for the spectral index and the tensor-to-scalar ratio from cosmological attractor models in the slow-roll regime. This model includes several constants with variable values. Therefore, we construct a family of models with different values of the constants. We consider the scalar power spectral amplitude and estimate possible values of the model parameters using modern observational data [13]

The paper is organized as follows. In Sect. 2, we reformulate the problem of the slow-roll regime in Einstein-GaussBonnet gravity in terms of e-folding numbers. In Sect. 3, we apply our reformulation to construct a model with variable values of parameters, which leads to the cosmological attractor approximation for inflationary parameters. To satisfy observational data we introduce a restriction to the model parameters. In conclusion, we summarize our results. 


\section{Slow-roll regime in Einstein-Gauss-Bonnet gravity}

We consider the model with the Gauss-Bonnet term multiplied by a function of the scalar field $\phi$ :

$S=\int d^{4} x \sqrt{-g}\left[\frac{R}{2}-\frac{\partial^{\nu} \phi \partial_{\nu} \phi}{2}-V(\phi)-\frac{\xi(\phi)}{2} \mathcal{G}\right]$,

where $\mathcal{G}=R_{\mu \nu \rho \sigma} R^{\mu \nu \rho \sigma}-4 R_{\mu \nu} R^{\mu \nu}+R^{2}$. The model is presented in Planckian units: $h=c=8 \pi G=1$. Application of the variation principe leads to the following system of equations [35] in spatially flat FLRW metric with $d s^{2}=-d t^{2}+a^{2}(t)\left(d x^{2}+d y^{2}+d z^{2}\right)$ :

$$
\begin{aligned}
& 6 H^{2}=\dot{\phi}^{2}+2 V+24 \dot{\xi} H^{3}, \\
& 2 \dot{H}=-\dot{\phi}^{2}+4 \ddot{\xi} H^{2}+4 \dot{\xi} H\left(2 \dot{H}-H^{2}\right), \\
& \ddot{\phi}+3 H \dot{\phi}+V_{, \phi}+12 \xi_{, \phi} H^{2}\left(\dot{H}+H^{2}\right)=0,
\end{aligned}
$$

where $H=\dot{a} / a$, the dot means the derivative of time: $\dot{A}=$ $d A / d t$. We consider the model (3) in FLRW metric in the slow-roll regime [35]:

$\dot{\phi}^{2} \ll V, \quad|\ddot{\phi}| \ll 3 H|\dot{\phi}|, \quad 4|\dot{\xi}| H \ll 1, \quad|\ddot{\xi}| \ll|\dot{\xi}| H$,

in which of the equations of motion are

$H^{2} \simeq \frac{V}{3}, \dot{H} \simeq-\frac{\dot{\phi}^{2}}{2}-2 \dot{\xi} H^{3}, \dot{\phi} \simeq-\frac{V_{, \phi}+12 \xi_{, \phi} H^{4}}{3 H}$

The slow-roll parameters are

$$
\begin{aligned}
& \epsilon_{1}=-\frac{\dot{H}}{H^{2}}, \quad \epsilon_{i+1}=\frac{\mathrm{d} \ln \left|\epsilon_{i}\right|}{\mathrm{d} \ln a}, \quad i \geq 1, \\
& \delta_{1}=4 \dot{\xi} H, \quad \delta_{i+1}=\frac{\mathrm{d} \ln \left|\delta_{i}\right|}{\mathrm{d} \ln a}, \quad i \geq 1 .
\end{aligned}
$$

To get a cosmological attractor generalization we consider the model in the slow-roll regime using the e-folding number representation and the designation $A^{\prime}=\mathrm{d} A / \mathrm{d} N$ :

$$
\left(\phi^{\prime}\right)^{2} \simeq \frac{V^{\prime}}{V}+\frac{4}{3} \xi^{\prime} V=\frac{\left(H^{2}\right)^{\prime}}{H^{2}}+4 H^{2} \xi^{\prime} .
$$

We present the slow-roll parameters in terms of $H^{2}$ and $\xi$ :

$$
\begin{aligned}
& \epsilon_{1}=\frac{1}{2} \frac{\left(H^{2}\right)^{\prime}}{H^{2}}, \\
& \epsilon_{2}=\frac{\left(H^{2}\right)^{\prime}}{H^{2}}-\frac{\left(H^{2}\right)^{\prime \prime}}{\left(H^{2}\right)^{\prime}}=2 \epsilon_{1}-\frac{\left(H^{2}\right)^{\prime \prime}}{\left(H^{2}\right)^{\prime}}, \\
& \delta_{1}=-4 H^{2} \xi^{\prime}, \\
& \delta_{2}=-\frac{\left(H^{2}\right)^{\prime}}{H^{2}}-\frac{\xi^{\prime \prime}}{\xi^{\prime}}=-2 \epsilon_{1}-\frac{\xi^{\prime \prime}}{\xi^{\prime}} .
\end{aligned}
$$

The slow-roll approximation requires $\left|\epsilon_{i}\right| \ll 1,\left|\delta_{i}\right| \ll 1$.

The question of the restrictions to inflation scenarios related with the speed of sound in Einstein-Gauss-Bonnet gravity was considered in [36]. There are wonderful properties of the slow-roll regime: the speed of sound is real. According to [37] the speed of sound square can be represented in the form $c_{A}^{2}=1+\Delta c_{A}^{2}$, where

$\Delta c_{A}^{2}=-\frac{2 \delta_{1}^{2} \epsilon_{1}}{3 \delta_{1}^{2}+2\left(2 \epsilon_{1}-\delta_{1}\right)\left(1+\delta_{1}\right)}$.

In the general case of the slow-roll regime

$\Delta c_{A}^{2} \simeq-\left(\delta_{1}^{2} \epsilon_{1}\right) /\left(2 \epsilon_{1}-\delta_{1}\right) \ll 1$.

If $2 \epsilon_{1} \approx \delta_{1}$, then $\Delta c_{A}^{2} \simeq-2 \epsilon_{1} / 3 \ll 1$. Thus, we can conclude that $c_{A}^{2}>0$ in the slow-roll regime.

In [35] the spectral index of scalar perturbations and the tensor-to-scalar ratio are represented in terms of the slow-roll parameters:

$$
\begin{aligned}
& n_{s}=1-2 \epsilon_{1}-\frac{2 \epsilon_{1} \epsilon_{2}-\delta_{1} \delta_{2}}{2 \epsilon_{1}-\delta_{1}}, \\
& r=8\left|2 \epsilon_{1}-\delta_{1}\right|,
\end{aligned}
$$

and the expression for the amplitude [34] in terms of inflationary parameters is as follows:

$A_{s} \simeq \frac{2 H^{2}}{\pi^{2} r}$

We simplify the expression for the spectral index of scalar perturbations (17) remembering that $\epsilon_{2}=-\epsilon_{1}^{\prime} / \epsilon_{1}, \delta_{2}=$ $-\delta_{1}^{\prime} / \delta_{1}$ and tensor-to-scalar ratio (18) using (11). So, the inflationary parameters can be represented in the following form:

$$
\begin{aligned}
& n_{s}=1-2 \epsilon_{1}+\frac{r^{\prime}}{r}, \\
& r=8\left|2 \epsilon_{1}-\delta_{1}\right|=8\left(\frac{\left(H^{2}\right)^{\prime}}{H^{2}}+4 H^{2} \xi^{\prime}\right)=8\left(\phi^{\prime}\right)^{2} .
\end{aligned}
$$

\section{Generalization of the cosmological attractor method}

According to the inflationary parameters of cosmologicalattractor models without the Gauss-Bonnet term (2) the spectral index includes only a logarithmic derivative of the tensorto-scalar ratio

$\frac{r^{\prime}}{r}=-\frac{2}{N+N_{0}}, \quad$ and $\quad n_{s} \approx 1+\frac{r^{\prime}}{r}$

in the leading order of $1 / N$ approximation. The model without the Gauss-Bonnet term and the exponential potential leading to (2) was considered in [38]. In the next subsection we generalize this model to Einstein-Gauss-Bonnet gravity.

\subsection{Exponential form}

To generalize the cosmological attractor approximation to inflationary models with Gauss-Bonnet term we compare 
(21) with (2):

$\frac{r}{8}=\frac{\left(H^{2}\right)^{\prime}}{H^{2}}+4 H^{2} \xi^{\prime}=\frac{3 C_{\alpha}}{2\left(N+N_{0}\right)^{2}}$.

For simplicity we suppose that all terms in this equation are proportional to $1 /\left(N+N_{0}\right)^{2}$ and get the same approximation of the slow-roll parameter $\epsilon_{1}$ in leading order in $1 / N$ :

$$
\begin{aligned}
& H^{2}=H_{0}^{2} \exp \left(-\frac{3 C_{\beta}}{2\left(N+N_{0}\right)}\right), \\
& \xi=\xi_{0} \exp \left(\frac{3 C_{\beta}}{2\left(N+N_{0}\right)}\right),
\end{aligned}
$$

where $C_{\beta}$ is a constant. We substitute (24), (25) into (23) and get

$\frac{r}{8}=\frac{3 C_{\beta}}{2\left(N+N_{0}\right)^{2}}\left(1-4 \xi_{0} H_{0}^{2}\right)$,

fixing a relation between $C_{\alpha}$ and $C_{\beta}$ :

$C_{\beta}=\frac{C_{\alpha}}{1-4 \xi_{0} H_{0}^{2}}, \quad H_{0}^{2} \neq \frac{1}{4 \xi_{0}}$.

Accordingly (21) the derivative of the field is related with the e-folding number:

$\left(\phi^{\prime}\right)^{2}=\frac{3 C_{\alpha}}{2\left(N+N_{0}\right)^{2}} ; \phi^{\prime}=\frac{\omega_{\phi} \sqrt{\frac{3 C_{\alpha}}{2}}}{N+N_{0}}, \omega_{\phi}= \pm 1$,

and thus

$$
\begin{aligned}
& \phi=\omega_{\phi} \sqrt{\frac{3 C_{\alpha}}{2}} \ln \left(\frac{N+N_{0}}{N_{\phi}}\right), \\
& N+N_{0}=N_{\phi} \exp \left(\omega_{\phi} \sqrt{\frac{2}{3 C_{\alpha}}} \phi\right) .
\end{aligned}
$$

Using (8), (24) and (30) we construct the family of models with the Gauss-Bonnet interaction and potential with variable parameter $C_{\alpha}$ :

$$
\begin{aligned}
& V=3 H_{0}^{2} \exp \left(-\frac{3}{2} \frac{C_{\beta}}{N_{\phi}} \exp \left(-\omega_{\phi} \sqrt{\frac{2}{3 C_{\alpha}}} \phi\right)\right), \\
& \xi=\xi_{0} \exp \left(\frac{3}{2} \frac{C_{\beta}}{N_{\phi}} \exp \left(-\omega_{\phi} \sqrt{\frac{2}{3 C_{\alpha}}} \phi\right)\right),
\end{aligned}
$$

leading to appropriate inflationary scenarios. This model is a generalization of the general relativity model obtained in [38].

We would like to compare inflationary parameters of the obtained model (32) with inflationary parameters of the following model:

$$
V=3 H_{0}^{2}\left(1-\frac{3 C_{\beta}}{4 N_{\phi}} \exp \left(-\omega_{\phi} \sqrt{\frac{2}{3 C_{\alpha}}} \phi\right)\right)^{2},
$$

$$
\xi=\xi_{0}\left(1-\frac{3 C_{\beta}}{4 N_{\phi}} \exp \left(-\omega_{\phi} \sqrt{\frac{2}{3 C_{\alpha}}} \phi\right)\right)^{-2}
$$

In this model the relation between e-folding numbers and field values is different from (30) and can be presented in the form

$\frac{N+N_{0}}{N_{\phi}}=\exp \left(\omega_{\phi} \sqrt{\frac{2}{3 C_{\alpha}}} \phi\right)-\frac{3}{4} \frac{C_{\beta}}{N_{\phi}} \omega_{\phi} \sqrt{\frac{2}{3 C_{\alpha}}} \phi$,

$\phi=-\omega_{\phi} \sqrt{\frac{3 C_{\alpha}}{2}}\left(\right.$ LambertW $\left.\left(-\frac{4 N_{\phi}}{3 C_{\beta}} \exp \left(-\frac{4 N}{3 C_{\beta}}\right)\right)+\frac{4 N}{3 C_{\beta}}\right)$.

Here we should note that, if $\omega_{\phi}=+1$, then

$\exp \left(\omega_{\phi} \sqrt{\frac{2}{3 C_{\alpha}}} \phi\right)-\frac{3}{4} \frac{C_{\beta}}{N_{\phi}} \omega_{\phi} \sqrt{\frac{2}{3 C_{\alpha}}} \phi \simeq \exp \left(\omega_{\phi} \sqrt{\frac{2}{3 C_{\alpha}}} \phi\right)$

in the large field expansion and Eq. (35) can be roughly approximated by (30).

\subsection{Inflationary parameters}

In this subsection, we get the expressions for the inflationary parameters in terms of the fields. The tensor-to-scalar ratio and the spectral index of scalar perturbations can be presented in the following form [35]:

$r=8 Q^{2}, \quad n_{s}=1-Q \frac{V_{\phi}}{V}+2 Q_{, \phi}$,

where $Q=V{ }_{, \phi} / V+4 \xi_{, \phi} V / 3$. We consider (32) and (34) to check the correspondence of the expression for the inflationary parameters. In the comparative analysis we suppose $N_{\phi}=3 C_{\beta} / 4$ and $\omega_{\phi}=1$ for simplicity and consider the models

$$
\text { 1. } \begin{aligned}
V & =3 H_{0}^{2} \exp \left(-2 \exp \left(-\sqrt{\frac{2}{3 C_{\alpha}}} \phi\right)\right), \\
\xi & =\xi_{0} \exp \left(2 \exp \left(-\sqrt{\frac{2}{3 C_{\alpha}} \phi}\right)\right)
\end{aligned}
$$

and

$$
\text { 2. } \begin{aligned}
\tilde{V} & =3 H_{0}^{2}\left(1-\exp \left(-\sqrt{\frac{2}{3 C_{\alpha}}} \phi\right)\right)^{2}, \\
\tilde{\xi} & =\xi_{0}\left(1-\exp \left(-\sqrt{\frac{2}{3 C_{\alpha}} \phi}\right)\right)^{-2} .
\end{aligned}
$$

We use (36) to calculate the inflationary parameters for the model (37),

$$
\begin{aligned}
& \text { 1. } \quad n_{s}-1= \\
& \frac{8\left(4 H_{0}^{2} \xi_{0}-1\right) \exp \left(-\sqrt{\frac{2}{3 C_{\alpha}}} \phi\right)\left(1+\exp \left(-\sqrt{\frac{2}{3 C_{\alpha}}} \phi\right)\right)}{3 C_{\alpha}}
\end{aligned}
$$




$$
r=\frac{64\left(4 H_{0}^{2} \xi_{0}-1\right)^{2}}{3 C_{\alpha}} \exp \left(-2 \sqrt{\frac{2}{3 C_{\alpha}}} \phi\right),
$$

and for the model(38)

$$
\begin{aligned}
& 2 . \quad \tilde{n}_{s}-1= \\
& \frac{8\left(4 H_{0}^{2} \xi_{0}-1\right) \exp \left(-\sqrt{\frac{2}{3 C_{\alpha}}} \phi\right)\left(1+\exp \left(-\sqrt{\frac{2}{3 C_{\alpha}}} \phi\right)\right)}{3 C_{\alpha}\left(1-\exp \left(-\sqrt{\frac{2}{3 C_{\alpha}}} \phi\right)\right)^{2}}, \\
& \tilde{r}=\frac{64\left(4 H_{0}^{2} \xi_{0}-1\right)^{2} \exp \left(-2 \sqrt{\frac{2}{3 C_{\alpha}}} \phi\right)}{3 C_{\alpha}\left(1-\exp \left(-\sqrt{\frac{2}{3 C_{\alpha}}} \phi\right)\right)^{2}} .
\end{aligned}
$$

In the case of a large field $\phi$ the expressions for $r$ and $\tilde{r}$ coincide up to second order, the expressions for $n_{s}$ and $\tilde{n}_{s}$ coincide up to first order of $\exp \left(-\sqrt{\frac{2}{3 C_{\alpha}}} \phi\right)$. The precision coincides with the sensibility of the cosmological attractor approximation (2). To satisfy the proposal's sensibility we can write

$$
\begin{gathered}
n_{s} \simeq 1+\frac{8\left(4 H_{0}^{2} \xi_{0}-1\right)}{3 C_{\alpha}} \exp \left(-\sqrt{\frac{2}{3 C_{\alpha}} \phi}\right), \\
r \simeq \frac{64\left(4 H_{0}^{2} \xi_{0}-1\right)^{2}}{3 C_{\alpha}} \exp \left(-2 \sqrt{\frac{2}{3 C_{\alpha}}} \phi\right) .
\end{gathered}
$$

According to (27) these relations can be represented in the forms

$$
\begin{aligned}
n_{s} & \simeq 1-\frac{2}{N_{\phi}} \exp \left(-\sqrt{\frac{2}{3 C_{\alpha}} \phi}\right), \\
r & \simeq \frac{12 C_{\alpha}}{N_{\phi}^{2}} \exp \left(-2 \sqrt{\frac{2}{3 C_{\alpha}}} \phi\right),
\end{aligned}
$$

which are fully in correspondence to (2).

\subsection{Restriction to the model parameters}

According to the Planck data [13] the values of the scalar spectral index and the restriction to the tensor-to-scalar ratio are $n_{s} \approx 0.965 \pm 0.004$ and $r<0.056$. The value of the scalar power spectrum amplitude is $A_{s} \approx 2 \cdot 10^{-9}$.

The considered inflationary models with the GaussBonnet interaction can be represented more precisely, namely, to satisfy condition $\epsilon_{1}(N \simeq 0) \approx 1$ we should suppose $N_{0}=\sqrt{3 C_{\beta} / 4}$. To follow the notations of [38] we should suppose $N_{0}=1$ and $C_{\beta}=4 / 3$.

According to (2) the highest value of the constant $C_{\alpha}$ is related with modern observations' restriction to the tensorto-scalar ratio $r$ and the value of e-folding number at the beginning of inflation. At the same time the start point of inflation defines the value of the spectral index of scalar perturbations. We numerically estimate the value of the model parameters using (2) and suppose that the inflation begins:
1. at $N \approx 55-N_{0}$ before the end of inflation: $n_{s} \approx 0.964$ and $0 \leq C_{\alpha}<14.1$;

2. at $N \approx 60-N_{0}$ before the end of inflation: $n_{s} \approx 0.967$ and $0 \leq C_{\alpha}<16.7$;

3. at $N \approx 65-N_{0}$ before the end of inflation: $n_{s} \approx 0.969$ and $0 \leq C_{\alpha}<19.6$.

To get an expression for the scalar power spectrum amplitude we substitute (23) and (24) into (19):

$$
\begin{aligned}
A_{s} & \simeq \frac{H_{0}^{2}\left(N+N_{0}\right)^{2}}{6 \pi^{2} C_{\alpha}} \exp \left(-\frac{3 C_{\beta}}{2\left(N+N_{0}\right)}\right) \\
& =\frac{H_{0}^{2}\left(N+N_{0}\right)^{2}}{6 \pi^{2} C_{\alpha}} \exp \left(-\frac{2 N_{0}^{2}}{N+N_{0}}\right),
\end{aligned}
$$

from which we have

$$
\frac{H_{0}^{2}}{C_{\alpha}}=\frac{6 \pi^{2} A_{s}}{\left(N+N_{0}\right)^{2}} \exp \left(\frac{2 N_{0}^{2}}{N+N_{0}}\right) .
$$

To estimate $H_{0}^{2} / C_{\alpha}$ we suppose $N_{0} \approx 1$ in three cases:

1. if the start point of inflation $N \approx 54$, then $H_{0}^{2} / C_{\alpha} \approx$ $4.09 \cdot 10^{-11}$

2. if the start point of inflation $N \approx 59$, then $H_{0}^{2} / C_{\alpha} \approx$ $3.40 \cdot 10^{-11}$

3. if the start point of inflation $N \approx 64$, then $H_{0}^{2} / C_{\alpha} \approx$ $2.90 \cdot 10^{-11}$.

To estimate the relation between the model parameters $\xi_{0}$ and $C_{\alpha}$ we use Eq. (27)

$\xi_{0}=\frac{1}{4}\left(\frac{1}{C_{\alpha}}-\frac{1}{C_{\beta}}\right)\left(\frac{H_{0}^{2}}{C_{\alpha}}\right)^{-1}=\frac{1}{4}\left(\frac{1}{C_{\alpha}}-\frac{3}{4}\right)\left(\frac{H_{0}^{2}}{C_{\alpha}}\right)^{-1}$

in three cases:

1. if the start point of inflation $N \approx 54$, then $\xi_{0} \approx$ $\left(C_{\alpha}^{-1}-3 / 4\right) 6.10 \cdot 10^{9}$;

2. if the start point of inflation $N \approx 59$, then $\xi_{0} \approx$ $\left(C_{\alpha}^{-1}-3 / 4\right) 7.35 \cdot 10^{9}$;

3. if the start point of inflation $N \approx 64$, then $\xi_{0} \approx$ $\left(C_{\alpha}^{-1}-3 / 4\right) 8.62 \cdot 10^{9}$.

The value of the parameter $\xi_{0}$ is positive if $C_{\alpha}>4 / 3$; is 0 if $C_{\alpha}=4 / 3$; is negative if $C_{\alpha}<4 / 3$.

\section{Conclusion}

We use the equations of Einstein-Gauss-Bonnet gravity in the Friedmann universe and inflationary parameters in terms of the e-folding number for the slow-roll regime. With the help of this formulation, we obtain gravity models with the 
Gauss-Bonnet term leading to analytical expressions of the inflationary parameters coinciding with inflationary parameters of cosmological attractor models in the leading order approximation. The model is a generalization to the cosmological attractor of exponential form initially proposed for general relativity [38]. We consider the possible expansion of our models for a large field. We calculate and compare the inflationary parameters for the two models' estimated order of accuracies for the large field expansion.

Within the framework of the model we obtain an analytical expression for the scalar power spectrum amplitude. We estimate the model constants using observation data for the value of scalar power spectrum amplitude, the spectral index of scalar perturbations and the tensor-to-scalar ratio. We plan to apply our approach to a consideration of more complicated models with Gauss-Bonnet term and use the effective potential proposed in [39]. For future refinement, it should be noted that the representation of $n_{s}$ up to second order in $1 / N$ may lead to better agreement with modern observations [13]. Also the consideration can be expand to another types of relation between of tensor-to-scalar ratio and e-folding number, for example $r \sim\left(N+N_{0}\right)^{-1}[40]$.

The paper is partly supported by RFBR Grant 18-5245016.

Data Availability Statement This manuscript has no associated data or the data will not be deposited. [Authors' comment: This is a Theoretical Research Project.]

Open Access This article is licensed under a Creative Commons Attribution 4.0 International License, which permits use, sharing, adaptation, distribution and reproduction in any medium or format, as long as you give appropriate credit to the original author(s) and the source, provide a link to the Creative Commons licence, and indicate if changes were made. The images or other third party material in this article are included in the article's Creative Commons licence, unless indicated otherwise in a credit line to the material. If material is not included in the article's Creative Commons licence and your intended use is not permitted by statutory regulation or exceeds the permitted use, you will need to obtain permission directly from the copyright holder. To view a copy of this licence, visit http://creativecomm ons.org/licenses/by/4.0/.

Funded by SCOAP ${ }^{3}$.

\section{References}

1. R. Brout, F. Englert, E. Gunzig, The creation of the universe as a quantum phenomenon. Ann. Phys. 115, 78 (1978)

2. A.A. Starobinsky, Phys. Lett. B 91, 99 (1980)

3. D. Kazanas, Astrophys. J. 241, L59 (1980)

4. K. Sato, MNRAS 195, 467 (1981)

5. A.H. Guth, Phys. Rev. D 23, 347 (1981)

6. A.D. Linde, Phys. Lett. B 108, 389 (1982)

7. A. Albrecht, P.J. Steinhardt, Phys. Rev. Lett. 48, 1220 (1982)

8. A.D. Linde, Phys. Lett. B 129, 177 (1983)
9. V.F. Mukhanov, G.V. Chibisov, JETP Lett. 33, 532 (1981)

10. V.F. Mukhanov, G.V. Chibisov, Pisma. Zh. Eksp. Teor. Fiz. 33, 549 (1981)

11. A.A. Starobinsky, Phys. Lett. B 117, 175 (1982)

12. A. Starobinsky, Sov. Astron. Lett. 9, 302 (1983)

13. Y. Akrami et al. [Planck], [arXiv:1807.06211 [astro-ph.CO]]

14. F.L. Bezrukov, M. Shaposhnikov, Phys. Lett. B 659, 703 (2008). [arXiv:0710.3755 [hep-th]]

15. A.O. Barvinsky, A.Y. Kamenshchik, A.A. Starobinsky, JCAP 0811, 021 (2008). [arXiv:0809.2104 [hep-ph]]

16. F.L. Bezrukov, A. Magnin, M. Shaposhnikov, S. Sibiryakov, JHEP 1101, 016 (2011). [arXiv:1008.5157 [hep-ph]]

17. M. Galante, R. Kallosh, A. Linde, D. Roest, Phys. Rev. Lett. 114(14), 141302 (2015). arXiv:1412.3797 [hep-th]

18. R. Kallosh, A. Linde, JCAP 1307, 002 (2013). arXiv:1306.5220 [hep-th]

19. E. Elizalde, S. Odintsov, E. Pozdeeva, S.Y. Vernov, JCAP 02, 025 (2016). arXiv:1509.08817 [gr-qc]

20. D.I. Kaiser, E.A. Mazenc, E.I. Sfakianakis, Phys. Rev. D 87, 064004 (2013). arXiv:1210.7487 [astro-ph.CO]

21. R.N. Greenwood, D.I. Kaiser, E.I. Sfakianakis, Phys. Rev. D 87, 064021 (2013). arXiv:1210.8190 [hep-ph]

22. M. Dubinin, E.Y. Petrova, E. Pozdeeva, M. Sumin, S.Y. Vernov, JHEP 12, 036 (2017). arXiv:1705.09624 [hep-ph]

23. R. Kallosh, A. Linde, JCAP 1312, 006 (2013). arXiv:1309.2015 [hep-th]

24. M. Dubinin, E.Y. Petrova, E. Pozdeeva, S.Y. Vernov, Int. J. Geom. Methods Mod. Phys. 15, 1840001 (2018). arXiv:1712.03072 [hep$\mathrm{ph}]$

25. E.O. Pozdeeva, M.R. Gangopadhyay, M. Sami, A.V. Toporensky, S.Y. Vernov, 'Inflation with a quartic potential in the framework of Einstein-Gauss-Bonnet gravity (2020). arXiv:2006.08027 [gr-qc]

26. I. Fomin, arXiv:2004.08065 [gr-qc]

27. S. Odintsov, V. Oikonomou, F. Fronimos, arXiv:2003.13724 [grqc]

28. N. Rashidi, K. Nozari, Astrophys. J. 890, 58. arXiv:2001.07012 [astro-ph.CO]

29. L. Granda, D. Jimenez, arXiv:1910.11289 [gr-qc]

30. K. Kleidis, V. Oikonomou, Nucl. Phys. B 948, 114765 (2019). arXiv: 1909.05318 [gr-qc]

31. S. Odintsov, V. Oikonomou, Phys. Rev. D 98(4), 044039 (2018). arXiv: 1808.05045 [gr-qc]

32. Z. Yi, Y. Gong, M. Sabir, Phys. Rev. D 98(8), 083521 (2018). arXiv: 1804.09116 [gr-qc]

33. S. Chakraborty, T. Paul, S. SenGupta, Phys. Rev. D 98(8), 083539 (2018). arXiv:1804.03004 [gr-qc]

34. C. van de Bruck, K. Dimopoulos, C. Longden , C. Owen, arXiv:1707.06839 [astro-ph.CO]

35. Z. Guo, D.J. Schwarz, Phys. Rev. D 81, 123520 (2010). [arXiv:1001.1897 [hep-th]]

36. G. Hikmawan, J. Soda, A. Suroso, F.P. Zen, Phys. Rev. D 93(6), 068301 (2016). arXiv:1512.00222 [hep-th]

37. S. Odintsov, V. Oikonomou, Phys. Lett. B 805, 135437 (2020). arXiv:2004.00479 [gr-qc]

38. V. Mukhanov, Eur. Phys. J. C 73, 2486 (2013). arXiv:1303.3925 [astro-ph.CO]

39. E.O. Pozdeeva, M. Sami, A.V. Toporensky, S.Y. Vernov, Phys. Rev. D 100(8), 083527 (2019). arXiv:1905.05085 [gr-qc]

40. S.D. Odintsov, V.K. Oikonomou, Viable Inflation in ScalarGauss-Bonnet Gravity and Reconstruction from Observational Indices. Phys. Rev. D 98(4), 044039 (2018). https://doi.org/10. 1103/PhysRevD.98.044039. arXiv:1808.05045 [gr-qc] 